\title{
Emotions in the Ether: Strategies for Effective Emotional Expression in Text-Messages
}

\section{Andrew Albritton, Missouri State University, USA}

\begin{abstract}
This essay offers an exploration of the practice of emotional expression in text-messages, and gives recommendations concerning how best to signify emotion when communicating with text-messages. Emotions can be shown in text-messages in two ways: With words and with orthography. Two potential problems associated with expressing emotions in text-messages are ambiguity of tone and disinhibited communicative behavior. Two of the advantages of expressing emotions in text-messages are ease of contact between interlocutors and the control text-message senders have over their messages. When the two methods of expressing emotion in text-messages are understood, the two problems are avoided, and the two benefits are embraced, text-messages can be more effective tools for sharing emotional meaning- and thus more effective tools for personal connection.
\end{abstract}

Keywords: emotional expression, text-messages 


\section{Emotions in the Ether: Strategies for Effective Emotional Expression in Text-Messages}

Text-messages are an enormously popular means of communicating, especially among young people (Lenhart et al., 2010; Smith, 2011). Because text-messages are so prevalent, it is only natural that they are used to transmit socioemotional meanings in addition to more prosaic informational meanings. Even in the days when text-messaging was relatively new, several scholars noted that European youths often used text-messages for emotive and relational purposes (Harkin, 2003; Kasesniemi \& Rautiainen, 2002; Ling \& Yttri, 2002). Such expressions of affective states, in any medium, are vital components of human communication. As Schnoebelen (2012) wrote in the abstract of his Ph.D. dissertation:

People use language to position themselves, their audiences, and their topics relative to one another. Expressions of emotions are more than internal states made visible, they are actions that have particular interpersonal causes and consequences, which are understood linguistically (“I'm mad/happy/scared") and which collectively add up. (p. iv)

In this essay, the nature of emotive expression in text-messages is explored. First, the methods of conveying emotion in text-messages are discussed. Second, some of the problems associated with communicating emotion in text-messages are reviewed, and some strategies to overcome these problems are given. And third, some of the benefits of using text-messages to express emotive meanings are highlighted.

It should be noted that any form of text-based mobile communication is being included in this discussion as a form of text-messaging. When using the Internet to transmit text-messages (via, for instance, WhatsApp or iMessage), many forms of communication, including pictures, videos, GIFs, and audio recordings, can be deployed, and these media can obviously be used to express emotion; but, in this essay, the discussion is being limited to those less extravagant methods of expression which can be easily constructed with the basic keyboards associated with most mobile phones (including emoji keyboards). Moreover, it should be mentioned that many of the principles discussed in the essay could extend to other popular forms of textual media such as email and Instant Messaging (IM). 


\section{Methods of Conveying Emotion in Text-Messages}

Emotion can be conveyed in a text-message in two ways. The first and most obvious way is through the words of the message. A writer can simply write, "This makes me angry" or "I love you". This first type of emotional representation may be termed Lexical Emotive Expression. The second way that a person can convey emotion in a text-message is through the non-lexical aspects of the message, which is to say, through spelling, spacing, capitalization, and punctuation marks (including, for our purposes, emoticons and emoji). This second type of emotional representation may be termed Orthographic Emotive Expression. This dichotomy, it will be noticed, is analogous to the dichotomy of the verbal and nonverbal forms of communication that we see in in-person discourse, a point that will be further discussed in the following section.

Lexical Emotive Expressions are fairly straightforward; if messages containing these types of expressions are delivered without technical hindrance, and if their recipients know the definitions of the words being used in them, effective communication should occur. Orthographic Emotive Expressions are a bit more interesting and can be constructed in a number of ways. Anecdotally, it seems that some intentional misspellings, as well as some playful forms of letter spacing, can indicate a sense of lightheartedness and amiability. Words written with spaces between letters can also be seen as giving emphasis to words by spreading them out on the screen (Crystal, 2001); incidentally, there may be, in such stretched words, a paralinguistic signification - i.e., such words may have the effect (or intended effect) of the recipient of the message "hearing" the word in his or her mind as elongated, just as if he or she was hearing the writer pronounce the word aloud in a dramatically lengthened manner. Writing words in all capital letters can give emphasis to words as well, and such writing is popularly understood to signify yelling (Arendholz, 2013); because of the latter perception, this orthographic method may be a way to convey negative affect. Punctuation marks are another means by which people can share emotive meaning. Exclamation points, in addition to showing excitement, can signify friendliness and serve as emphasizers of different types of messages (Waseleski, 2006). Punctuation strings of varying kinds (from strings of exclamation points to strings of question marks to strings of one or more exclamation points with one or more question marks) can show excitement and serve as emphasizers as well (Kalman \& Gergle, 2009). Some young people view the period as a device that shows harshness or terseness (Baron \& Ling, 2011); while the period is not always used in such a negative way - it is, after all, the 
standard way to end a noninterrogative and non-exclamatory sentence - the deployment of this mark may well be a way, in certain circumstances, to convey flatness, causticness, or an end to a conversation. Emoticons, as their names would suggest, can also be used as signs of emotion. The :) can, for instance, demonstrate happiness or some other form of positive affect, while, contrastingly, the : ( can stand for sadness or some other form of negative affect. It should be noted, however, that emoticons can also serve the function of clarifying the social action being performed by the author of a message, as in the case of a person who demonstrates that he or she is joking with one of the tongue-sticking-out emoticons :p (Dresner \& Herring, 2010). The premade pictographs known as emoji can function similarly to emoticons, although the hundreds of emoji available allow for more elaborate constructions. This discussion of orthographic ways to share emotion is not exhaustive, but rather is intended to illustrate the point that emotive meanings can be conveyed in ways above and beyond the words of a message.

\section{Problems Associated with Emotive Expression in Text-Messages}

The scholarly literature indicates at least two potential problems associated with emotive expression in text-messages. The first is the potential for text-messages to be tonally opaque (Kelly et al., 2012). Humans frequently communicate logical meanings with words and emotional meanings with nonverbal and paralinguistic behavior (Richmond, McCroskey, \& Payne, 1991). Our facial expressions, bodily movements, and vocalic expressions can be nuanced and eloquent - and such articulate forms of communication are lacking in purely textual messages. Without proper construction, the emotions underlying text-messages can be unclear, and this emotional nebulousness can lead to confusion (Grinter \& Eldridge, 2001) and even interpersonal discord (Lenhart et al., 2010). There are a number of steps that can be taken to ensure that such problems of ambiguity do not arise. In the first place, in contexts in which emotions are an important part of the message, it may be best to forego the textmessage medium in favor of face-to-face interaction, whether in-person or via video: When interlocutors can see and hear each other, the full repertoire of nonverbal and paralinguistic cues can be employed, and certain emotive meanings may be more effectively conveyed. At the very least, a phone call, in which paralinguistic behavior would be audible, might be a better option than a textmessage. In those cases where a text-message would be appropriate, or is perhaps the only way to effectively or efficiently communicate with an interlocutor, and yet there could be some injurious effect because of the lack of tone, it should be remembered that Lexical Emotive 
Expressions are always an option: Text-message writers can always simply write, in words, what they are feeling. But even messages such as these can seem to be insincere: Consider for example the message "Wow. That's great. So happy for you." Some people may take this message as too flat, or even as sarcastic, because of the use of periods (Baron \& Ling, 2011). When there is the potential for this type of misunderstanding, writers can combine Lexical Emotive Expressions with appropriate Orthographic Emotive Expressions. The preceding hypothetical message, when given some apt Orthographic Emotive Expressions, could read, "Wow! That's great!! So happy for you)". When both types of expression are placed in a message - when, that is, people state their emotions and give, if appropriate to the communicative context, apposite orthographic indications of the stated emotions - there is less likelihood of tonal opacity, and such messages will thus be better understood and more efficacious.

The second problem associated with emotive expression in text-messages is the potential for disinhibition (Harrison \& Gilmore, 2012). Because text-messages are (usually) sent to people who are not physically present, message senders may not feel the social constraints they would feel if the recipient were present, and they may, therefore, feel freer to send hurtful or otherwise negative messages. Harrison and Gilmore (2012) studied the text-messaging practices of American college students and reported the following: "Most people in our sample use texting for maleficence. Also, over a quarter admit to texting someone after being told to stoparguable instances of cyberbullying" (p. 516). Given the possibility for disinhibition, it is advisable that people not send messages when they are swept up in tides of negative emotion; the messages produced under such circumstances could cause considerable harm. Once the emotion has ebbed away, senders can approach the communicative situation with more equanimity and rationality, and they can therefore make better decisions about the messages they compose. It may be helpful for message senders to consider whether or not they would say, in person, what they are typing. If they would not, then it is probably best not to send the text-message.

\section{Benefits of Using Text-Messages to Send Emotive Meanings}

While there are some potential problems with conveying emotive meanings in text-messages, there are some evident advantages to doing so as well. For one, text-messages allow people to stay in contact with one another very easily. A text-message can be sent when interlocutors are 
separated from one another - perhaps by a great distance - and when a voice or video call cannot, for whatever reason, be made. Thus text-messages allow for emotional expression and support in situations when people might not otherwise be able to contact each other. Moreover, there is a certain degree of directness to text-messages, since they are delivered directly to interlocutors' phones; and yet, at the same time, text-messages are not importuning - they do not necessarily call for the recipient's immediate and undivided attention (although, of course, the message sender may hope for, or even request in the message, instantaneous attention from the recipient).

A second advantage to emotive expression in text-messages is the great control that textmessage writers have over their digital missives. Text-message senders have the opportunity to carefully think about the construction of their messages and to revise them before sending them. The opportunities for reflection and editing afforded by the text-message medium can be of tremendous benefit when conveying any emotional meanings - but they can be especially helpful when composing messages in which the writer desires to communicate emotions that are difficult to express, nuanced, or complicated. This benefit of textmessaging was posited cogently in Walther's (2007) hyperpersonal model (which, it should be noted, pertains to communication via written digital messages in general, and not only text-messages). Walther (1997) first introduced Social Information Processing Theory, which posited that people can use digital media as a means of developing intimate relationships, but such relationships take more time to cultivate, since, in the digital sphere, relatively small amounts of information must be passed back and forth between people over time. Walther (2007) then developed these ideas further in the hyperpersonal model, which highlights some of the positive aspects of communication via digital media. Those benefits include the following: Digital messages are editable; people can take time to think about their messages; digital messages preclude the possibility of an interlocutor picking up on nonverbal cues the messages sender would not want to be perceived; and composing messages in a digital media allows for senders to give their full mental attention to the task of writing. As Walther (2007) wrote, "When CMC [computer-mediated communication] users are motivated to do so, these processes allow them to manage impressions and ultimately exceed parallel $\mathrm{FtF}$ [face-to-face] partnerships in social orientation or intimacy, according to the hyperpersonal perspective" (p. 2541). In this view, text-message conversations have the possibility of being even better, in some ways, than inperson conversations. In particular, it is worth drawing attention to the fact that text-messages 
afford the opportunity for a conversation in which nonverbal behavior is not seen and paralinguistic behavior is not heard. Above, it was shown that the absence of nonverbal and paralinguistic cues can be detrimental to emotive communication in textmessages - but Walther (2007) astutely reminds us that the absence of these cues is sometimes not only acceptable, but desirable. There are situations in which a person is overwhelmed with a certain emotion, and yet he or she does not want that emotion to be perceived, or does not want the strength of the emotion to be perceived. In an in-person exchange, covering up emotions can be hard, particularly if those emotions are powerful. In text-messages, on the other hand, it is relatively simple to avoid showing emotions an interlocutor does not want to show. Similarly, sometimes a person may feel, and wish to express, a given emotion, but, because of fatigue or other conflicting emotions, may have trouble expressing that emotion effectively in an in-person conversation; in a text-message, the person could use Lexical Emotive Expressions or Orthographic Emotive Expressions or both to effectually convey the emotion(s) he or she wishes to convey.

\section{Conclusion}

People can share emotions in text-messages with Lexical Emotive Expressions and Orthographic Emotive Expressions. There are certain problems associated with using textmessages to convey emotion that text-message senders should avoid. The first problem is undesired emotional ambiguity; this difficulty can be overcome in many cases if people speak in person (or via phone or video), or if they construct their messages with appropriate Lexical Emotive Expressions and Orthographic Emotive Expressions. The second problem is disinhibition; this pitfall can be avoided if interlocutors will take the time to carefully reflect on the appropriateness and potential effects of a text-message; moreover, message senders can consider whether they would be willing to utter a potential message aloud if face-to-face with the intended recipient of the message - if they would not, then the message may not be one they should send. There are also benefits to using text-messages to convey emotive meaning. The first is ease of contact. The second is the control over text-messages that message senders have. When people embrace the advantages of emotive text-messaging, and employ the strategies outlined above for overcoming the disadvantages associated with emotive texting, they can engage in more effective and enjoyable digital conversations. 


\section{References}

Arendholz, J. (2013). (In)Appropriate online behavior: A pragmatic analysis of message board relations. Amsterdam: John Benjamins.

Baron, N., \& Ling, R. (2011).Necessary smileys \& useless periods.Visible Language, 45(1/2), 45-67.

Crystal, D. (2001). Language and the Internet. Cambridge: Cambridge University Press.

Dresner, E. \& Herring, S. C. (2010). Functions of the nonverbal in CMC: Emoticons and illocutionary force. Communication Theory, 20(3), 249-268.

Grinter, R. E., \& Eldridge, M. (2001).y do tngrs luv 2 txt msg?. In W. Prinz, M. Jarke, Y. Rogers, K. Schmidt \& V. Wulf (Eds.), Proceedings of the Seventh European Conference on Computer-Supported Cooperative Work ECSCW '01, Bonn, Germany (219-238). Dordrecht, Netherlands: Kluwer Academic Publishers.

Harkin, J. (2003). Mobilisation: The growing public interest in mobile technology. London: Demos.

Harrison, M. A., \& Gilmore, A. L. (2012). U txt WHEN? College students's social contexts of text messaging.Social Science Journal, 49(4), 513-518.

Kalman, Y., \&Gergle, D. (2009). Letter and punctuation mark repeats as cues in computermediated communication.95th annual meeting of the National Communication Association in Chicago, IL. Retrieved from http://www.kalmans.com/NCA09Cues.pdf

Kasesniemi, E., \& Rautiainen, P. (2002).Mobile culture of children and teenagers in Finland. In J. E. Katz \& M.A. Aakhus (Eds.), Perpetual Contact: Mobile Communication, Private Talk, Public Performance (pp. 170-192). Cambridge: Cambridge University Press.

Kelly, L., Keaten, J. A., Becker, B., Cole, J., Littleford, L., \& Rothe, B. (2012). It's the American lifestyle!: An investigation of text messaging by college students. Qualitative Research Reports in Communication, 13(1), 1-9.

Lenhart, A., Ling, R., Campbell, S., \& Purcell, K. (2010).Teens and mobile phones. Retrieved from http://www.pewinternet.org/Reports/2010/Teens-and-MobilePhones.aspx

Ling, R., \& Yttri, B. (2002). Hyper-coordination via mobile phones in Norway. In J. E. Katz \& M. A. Aakhus (Eds), Perpetual contact: Mobile communication, private talk, public performance (pp. 139-169). Cambridge: Cambridge University Press. 
Richmond, V. P., McCroskey, J. C., \& Payne, S. K. (1991). Nonverbal behavior in interpersonal relations. Englewood Cliffs, NJ: Prentice-Hall.

Schnoebelen, T. J. (2012). Emotions are relational: Positioning and the use of affective linguistic resources. (Doctoral dissertation). Stanford University, Stanford, California, USA. Retrieved from http://stanford.edu/ tylers/notes/papers/emotion/Dissertation_Schnoebelen_final_82912.pdf

Smith, A. (2011). Americans and text messaging: Summary of findings. Retrieved from http://www.pewinternet.org/Reports/2011/Cell-Phone-Texting-2011/SummaryofFindings/Summary-of-Findings.aspx

Walther, J. B. (1997). Group and interpersonal effects in international computer-mediated collaboration. Human Communication Research, 23(3), 342-369.

Walther, J. B. (2007). Selective self-presentation in computer-mediated communication: Hyperpersonal dimensions of technology, language, and cognition. Computers in Human Behavior, 23(5), 2538-2557.

Waseleski, C. (2006). Gender and the use of exclamation points in computer-mediated communication: An analysis of exclamations posted to two electronic discussion lists. Journal of Computer-Mediated Communication, 11(4), 1012-1024. 